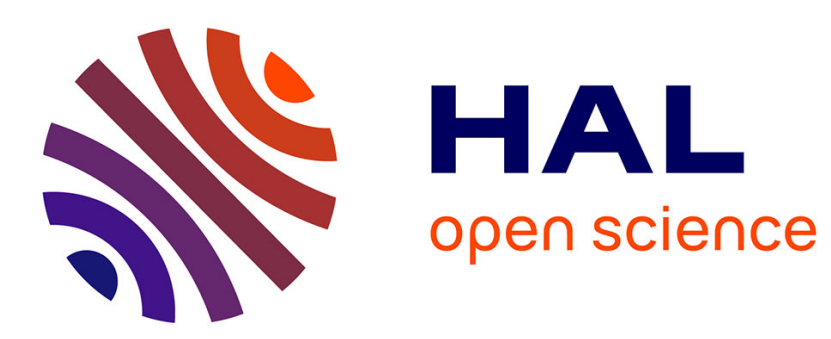

\title{
Perpendicular spin-torque switching with a synthetic antiferromagnetic reference layer
}

\author{
Ioan Tudosa, J. A. Katine, S. Mangin, Eric E. Fullerton
}

\section{To cite this version:}

Ioan Tudosa, J. A. Katine, S. Mangin, Eric E. Fullerton. Perpendicular spin-torque switching with a synthetic antiferromagnetic reference layer. Applied Physics Letters, 2010, 96, 10.1063/1.3441402 . hal-02099438

\section{HAL Id: hal-02099438 \\ https://hal.univ-lorraine.fr/hal-02099438}

Submitted on 24 Apr 2019

HAL is a multi-disciplinary open access archive for the deposit and dissemination of scientific research documents, whether they are published or not. The documents may come from teaching and research institutions in France or abroad, or from public or private research centers.
L'archive ouverte pluridisciplinaire HAL, est destinée au dépôt et à la diffusion de documents scientifiques de niveau recherche, publiés ou non, émanant des établissements d'enseignement et de recherche français ou étrangers, des laboratoires publics ou privés. 


\section{Perpendicular spin-torque switching with a synthetic antiferromagnetic reference layer}

Cite as: Appl. Phys. Lett. 96, 212504 (2010); https://doi.org/10.1063/1.3441402

Submitted: 18 December 2009 . Accepted: 08 May 2010 . Published Online: 27 May 2010

Ioan Tudosa, J. A. Katine, S. Mangin, and Eric E. Fullerton

\section{ARTICLES YOU MAY BE INTERESTED IN}

Spin torque switching of perpendicular Ta $|\mathrm{CoFeB}| \mathrm{MgO}$-based magnetic tunnel junctions

Applied Physics Letters 98, 022501 (2011); https://doi.org/10.1063/1.3536482

Very strong antiferromagnetic interlayer exchange coupling with iridium spacer layer for perpendicular magnetic tunnel junctions

Applied Physics Letters 110, 092406 (2017); https://doi.org/10.1063/1.4977565

Perpendicular magnetic tunnel junctions with synthetic antiferromagnetic pinned layers

based on $[\mathrm{Co} / \mathrm{Pd}]$ multilayers

Journal of Applied Physics 113, $17 B 909$ (2013); https://doi.org/10.1063/1.4799974

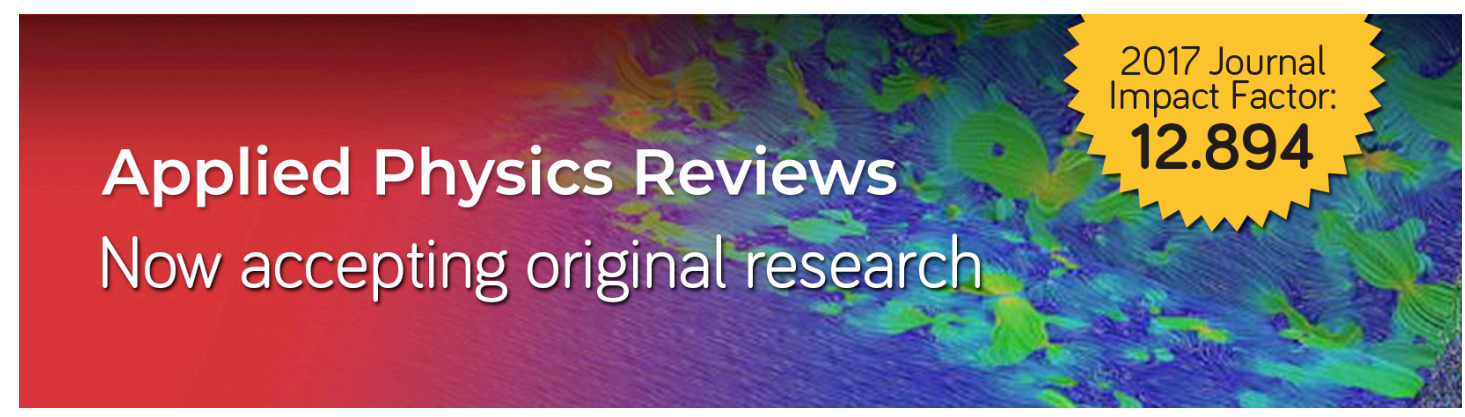

Appl. Phys. Lett. 96, 212504 (2010); https://doi.org/10.1063/1.3441402

96, 212504

(c) 2010 American Institute of Physics. 


\title{
Perpendicular spin-torque switching with a synthetic antiferromagnetic reference layer
}

\author{
Ioan Tudosa, ${ }^{1, a)}$ J. A. Katine, ${ }^{2}$ S. Mangin, ${ }^{3}$ and Eric E. Fullerton ${ }^{1, b)}$ \\ ${ }^{1}$ Center for Magnetic Recording Research, University of California-San Diego, La Jolla, \\ California 92093-0401, USA \\ ${ }^{2}$ San Jose Research Center, Hitachi Global Storage Technologies, 3403 Yerba Buena Road, San Jose, \\ California 95135, USA \\ ${ }^{3}$ Institut Jean Lamour, CNRS UMR 7198, Nancy-Université, B.P. 239 F-54506, Vandouvre lès Nancy, \\ France
}

(Received 18 December 2009; accepted 8 May 2010; published online 27 May 2010)

\begin{abstract}
Spin-torque switching of perpendicular anisotropy nanopillar spin valve devices with synthetic antiferromagnetic reference layers is presented. The use of composite reference layer reduces the dipolar interaction with the free layer yielding two remanent states. We derive a complete current-field phase diagram including minor loops of the free layer where we determine the relative dipolar fields and effective spin polarization of the reference layer in both the parallel and antiparallel configurations. We find the magnetic response of the reference layer relatively insensitive to the spin polarized current which may prove advantageous for spin-torque applications. (C) 2010 American Institute of Physics. [doi:10.1063/1.3441402]
\end{abstract}

Magnetic nanopillars made from a spin valve structure (two ferromagnetic layers separated by a nonmagnetic layer) are candidates for spintronic memory devices ${ }^{1,2}$ with recent focus on devices where a spin-polarized current is used to reverse the relative magnetization orientation of the layers. ${ }^{3-5}$ For most of the applications being considered, reductions in the critical currents for magnetization reversal are still needed. ${ }^{3}$ Devices with perpendicular magnetic anisotropy ${ }^{6-10}$ are one approach for reducing the critical currents, as the required current is directly proportional to the thermal stability of the free layers. ${ }^{7}$ However, in perpendicular anisotropy devices the strong dipolar interaction between the reference and free layer biases the free layer. With decreasing device size this bias is sufficient so that for zero applied field only one magnetic state is accessible ${ }^{5}$ limiting memory applications. In addition, experimental studies of the current-field phase diagrams of magnetization switching in perpendicular magnetic anisotropy nanopillars has yielded results that differ from expectations based on simple macrospin models which may result, in part, from dipolar interaction between the layers. ${ }^{6}$ In order to address these issues we present the experimental current-field phase diagram for perpendicularanisotropy nanopillar devices with synthetic antiferromagnetic (AF) reference layers that significantly reduce the dipolar interactions between the layers.

In the macrospin approximation at zero temperature the critical current for spin transfer reversal of the free layer is given by

$$
I_{C 0}=\left(\frac{2 e}{\hbar}\right) \frac{\alpha M_{S} V}{g(\theta) p} H_{\mathrm{eff}},
$$

where $M_{S}$ and $V$ are the saturation magnetization and volume of the free layer, $\alpha$ is its damping constant, $p$ is the spin polarization of the current, with $g(\theta)$ expressing the depen-

\footnotetext{
a) Current address: Avalanche Technology, 48371 Fremont Blvd., Ste. 101, Fremont, California 94538, USA

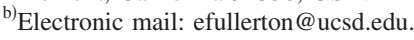

dence on the relative orientation angle of the magnetization vectors. ${ }^{11}$ In the case of perpendicularly magnetized layers the effective field acting on the free layer $H_{\text {eff }}=\left(H_{K}-4 \pi M_{S}\right.$ $\left.+H_{\text {app }}+H_{\text {dip }}\right)$ has contributions from magnetic anisotropy field $H_{K}$, demagnetizing field $-4 \pi M_{S}$, applied field $H_{\text {app }}$, and the dipolar field from the reference layer $H_{\text {dip. }}{ }^{6,7}$ To make a meaningful comparison of intrinsic properties of different materials, the switching currents when $H_{\text {app }}+H_{\text {dip }}=0$ should be compared. One can apply an external field to balance the dipole field but for device application it is preferred to engineer the reference layer to reduce $H_{\mathrm{dip}}$. For in-plane magnetized devices this is most commonly done using a synthetic-AF reference layer made of two AF-coupled magnetic layers to compensate the dipole fields. ${ }^{3,12}$ This has the additional benefit that the reduced net moment of the reference layer makes it less sensitive to perturbation coming from external magnetic fields.

The nanopillar structures used in our studies are $([\mathrm{Co} / \mathrm{Pd}] \times 4 / \mathrm{Co}) / \mathrm{Ru}([\mathrm{Co} / \mathrm{Pd}] \times 2 /[\mathrm{Co} / \mathrm{Ni}] \times 2 / \mathrm{Co}) /$ $\mathrm{Cu} /([\mathrm{Co} / \mathrm{Ni}] \times 2 /[\mathrm{Co} / \mathrm{Pd}])$. The layer thicknesses for each component are $\mathrm{Co}(3 \AA) / \mathrm{Pd}(7 \AA), \quad \mathrm{Co}(1.5 \AA) / \mathrm{Ni}(6 \quad \AA)$, $\mathrm{Co}(3 \AA), \operatorname{Ru}(9 \AA)$, and $\mathrm{Cu}(40 \AA)$. The films were grown by dc magnetron sputtering onto $\mathrm{Si}(100)$ wafers with a $2000 \AA$ thermal oxide and a $\mathrm{Ta}(30 \AA) / \mathrm{Cu}(350 \AA) / \mathrm{Pd}(30 \AA)$ buffer layer and were capped with a $\mathrm{Cu}(150 \AA) / \mathrm{Ta}(30 \AA)$ layer. The reference layer structure consists of the two magnetic layers AF-coupled by a thin Ru layer, where the bottom one is made of $\mathrm{Co} / \mathrm{Pd}$ multilayer only for higher anisotropy while the upper reference layer has a composite $[\mathrm{Co} / \mathrm{Pd}] /[\mathrm{Co} / \mathrm{Ni}]$ multilayer to enhance the polarization acting at the free layer. ${ }^{6}$ Magnetometry measurements of the as-grown films find the reference layer is fully compensated with a small AF coupling $(\sim 60 \mathrm{Oe})$ between the top reference layer and the free layer. The nanopillars were patterned by e-beam lithography in shapes of ellipses or rectangles, as described elsewhere. ${ }^{6}$ The device described in detail here has the shape of an ellipse with dimensions of $100 \times 210 \mathrm{~nm}^{2}$, however similar results were observed in other shaped devices. All of 


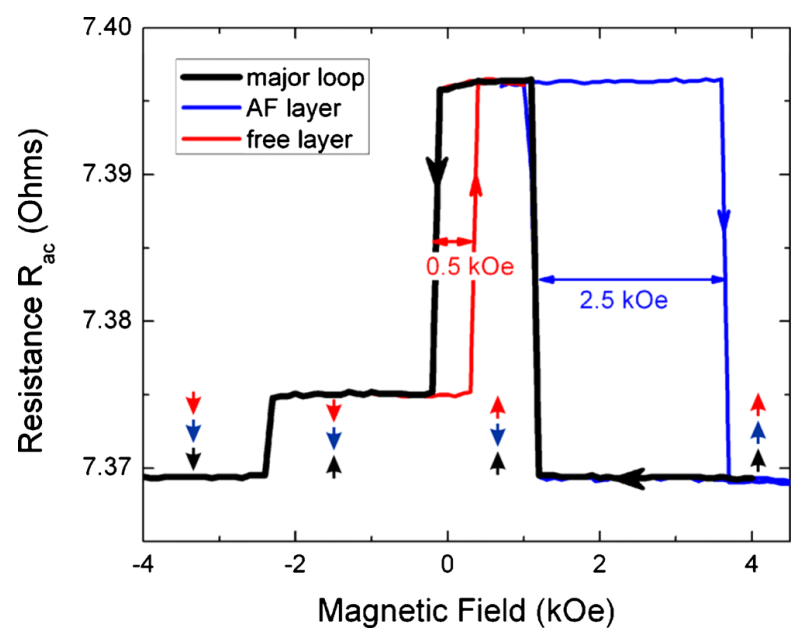

FIG. 1. (Color online) MR results for a $100 \times 210 \mathrm{~nm}^{2}$ nanopillar with a synthetic AF reference layer. The major loop is measured from positive to negative saturation fields applied perpendicular to the film planes. The arrows show the orientation of the magnetization in the three layers where the top arrow is the free layer. Minor loops for free layer and the top antiferromagnetically coupled reference layer are shown.

the magnetotransport measurements are quasistatic, performed using an ac-resistance bridge (excitation current of $10 \mu \mathrm{A} \mathrm{rms}$, at $13.7 \mathrm{~Hz}$ ) yielding the differential resistance $R_{\mathrm{ac}}=d V / d I$.

The ac-resistance as a function of the applied magnetic field is shown in Fig. 1 without a bias current. As the field is lowered from positive saturation where the magnetization of all the layers are parallel to the applied field, first the softer of the two reference layers (the one closer to the free layer) changes direction due to the $\mathrm{AF}$ exchange coupling through the $\mathrm{Ru}$ spacer layer. At this point all neighboring magnetic layers are antiparallel to each other and the resistance increases to its highest value. When further lowering the field, the free layer reverses in small negative fields becoming parallel with the top reference layer. This reduces the magnetoresistance (MR) to an intermediate level because of the MR signal from the AF-aligned reference layers. The last resistance change comes about as the harder reference layer is reversed by the field yielding negative saturation. The resistance change coming from the reference layers separated by the $\mathrm{Ru}$ spacer is roughly a third of the change coming from the two magnetic layers separated by the $\mathrm{Cu}$ spacer. The smaller effect is due to the smaller spin-diffusion length in $\mathrm{Ru}$ (Ref. 13) and the lower polarization efficiency of $(\mathrm{Co} /$ Pd) multilayers compared to $(\mathrm{Co} / \mathrm{Ni}){ }^{6}$

Also shown in Fig. 1 are the minor loops of the free layer and of the softer of the two reference layers. The free layer is bistable in zero applied field, with a free layer coercive field $H_{C}=250 \mathrm{Oe}$ and a shift of the loop of $100 \mathrm{Oe}$ arising from a ferromagnetic (relative to the top reference layer) coupling to the reference layer. Even though the reference layer moments are fully compensated a small dipolar field is expected on the free layer resulting from the closer proximity of the upper reference layer. However, the measured coupling for the AF aligned reference layer is somewhat larger than expected from micromagnetic calculations which may result from slight differences in patterning of the different layers. However, by adjusting the moment and thickness of the reference layers, it should be possible to
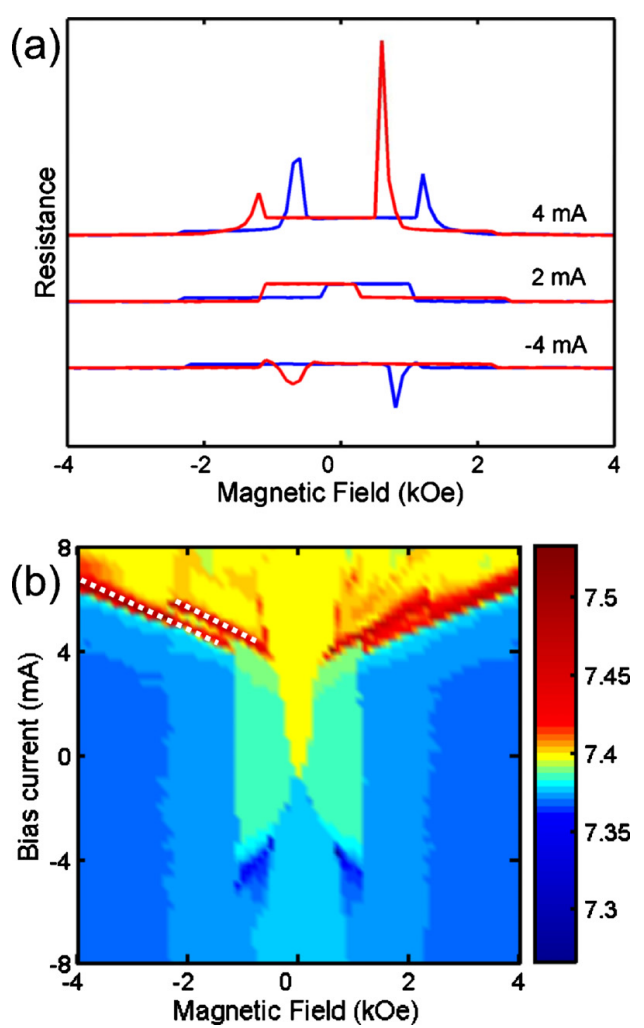

FIG. 2. (Color online) Current-field phase diagram for the nanopillar device shown in Fig. 1 with synthetic-AF reference layer. (a) MR curves for positive and negative magnetic field sweeps for several values of the bias current. (b) The average of the field sweeps at each bias current in a phase diagram. The code gives the average device ac-resistance. The dashed lines highlight the lateral displacement of the excitation depending on whether reference layer is parallel and antiparallel aligned.

further reduce the contributions of the reference layers. The minor loop of the top reference layer has a coercive field of $1.25 \mathrm{kOe}$ and is shifted $2.5 \mathrm{kOe}$ from zero field due the AF coupling to the lower reference layer.

In zero magnetic field, $H_{\text {app }}+H_{\text {dip }}=0$, the critical currents $I_{C}$ required to switch the magnetization from low resistance state (parallel alignment) to high resistance state (antiparallel alignment) and back are $1.5 \mathrm{~mA}$ and $-1.2 \mathrm{~mA}$, respectively. The ratio $I_{C} /\left(V H_{C}\right)$ depends primarily on the material parameters $\alpha, g(\theta)$, and $p$ assuming that $H_{C}$ scales with the anisotropy field. ${ }^{7}$ With the volume of free layer $V=52.5 \times 10^{-18} \mathrm{~cm}^{3}$, we find that $I_{C} /\left(V H_{C}\right)$ is 11.4 $\times 10^{13} \mathrm{~A} / \mathrm{kOe} \mathrm{cm}^{3}$, about 2.4 times less efficient in switching than the devices reported recently. ${ }^{7}$ This efficiency decrease is consistent with recent results on in-plane anisotropy devices that found a reduced effective polarization for synthetic-AF reference layers. ${ }^{14}$

An overview of the device behavior can be obtained through a current-field phase diagram by measuring the MR curves for different injected currents. After the device is saturated in a large magnetic field $(+6.5 \mathrm{kOe})$, the MR is measured as the field is varied from positive saturation to negative saturation and then back [example curves are shown in Fig. 2(a)]. The sum of the resistance obtained for both field directions is then plotted as the current-field phase diagram presented in Fig. 2(b). The phase diagram shows regions of hysteretic switching of both the free and reference layers as 


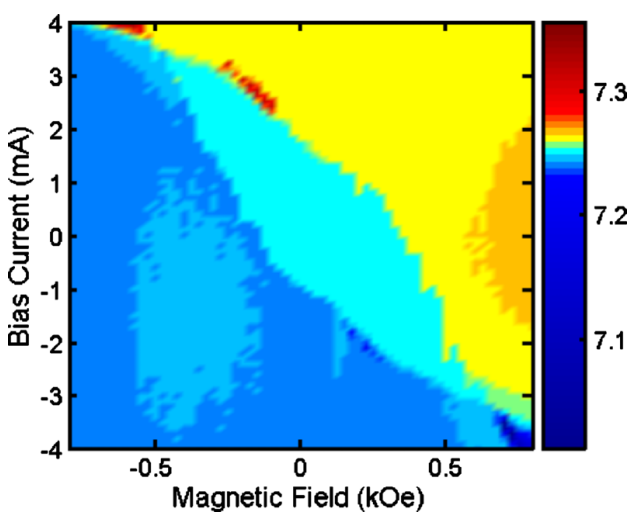

FIG. 3. (Color online) Current-field phase diagram for minor loops of free layer showing regions where the free layer is parallel and antiparallel to the top reference layer as well the hysteretic switching between the two states.

well as peaks in the ac resistance associated with the magnetic excitations of the free layer. ${ }^{15}$

In Fig. 3 we plot the phase diagrams for minor loops for just the free layer which is qualitatively similar to that published in Ref. 7, where the reversal of the free layer is sensitive to the applied current. In contrast, the field-induced reversal of the reference layers are relatively insensitive to the current. The vertical lines at $+/-1.2$ kOe in Fig. 2(b) correspond to the reversal of the top reference layer while the lines at $+/-2.2 \mathrm{kOe}$ result from the lower reference layer (Fig. 1). As can be seen from Fig. 2(b), these reversal fields are nearly independent of current over the entire current range of the experiment. The only deviation is for the top reference at large negative currents $<-4 \mathrm{~mA}$. At these currents we no longer see the reversal of the free layer. The large negative current ferromagnetically couples the free and top reference layer such that they reverse as a unit at lower fields, $+/-0.8 \mathrm{kOe}$. Although not shown, these vertical lines (at $+/-0.8$ and $+/-2.2 \mathrm{kOe}$ ) persist for negative currents as high as $-18 \mathrm{~mA}$. The fact that the AF reference layers are insensitive to spin-torque excitations makes them suitable for spin-torque devices. While the origin of this effect is not clear, recent experiments and modeling of in-plane synthetic $\mathrm{AF}$ layers find that they are less sensitive to spin-torque excitations. ${ }^{16,17}$

For large positive currents we observe peaks in $R_{\mathrm{ac}}$ that arise from precessional modes of the free layer. The critical current scales linearly with applied field (with a slope of 0.8 $\mathrm{mA} / \mathrm{kOe}$ ) as seen previously ${ }^{15}$ and expected from Eq. (1). For large applied field $(>2.2 \mathrm{kOe}$ or $<-2.2 \mathrm{kOe})$ the reference layers are ferromagnetically aligned. However, there are regions of the phase diagram $[(+/-1.2)-2.2$ kOe field ranges] where the precession modes of the free layer exist both when the reference layers are parallel and antiparallel for the same applied field. As the reference layers switch from parallel to antiparallel there is both a change in the dipolar magnetic field acting on the free layer and a possible change in the effective spin polarization from the free layer. The change in dipolar field leads to a lateral displacement of the excitation that results in two roughly parallel lines. These lines are highlighted as dashed lines in Fig. 2(b). The slopes of the lines are given by the prefactor in Eq. (1). There is a slight 10\%-15\% increase in the slope comparing the reference layer being antiparallel to parallel indicating a commensurate increase in the effective spin-polarization for the parallel reference layers. The displacement gives an estimation of the dipolar field from the switched reference layer acting on the free layer. For this device the excitations are displaced about 600 Oe by changing from parallel to AF alignments of the reference layers. However, this slightly overestimates the dipolar field change because of the commensurate change in the effective spin polarization.

In conclusion, we fabricated spin valve nanopillar devices with a synthetic AF reference layer structure that minimizes the dipolar field acting on the free layer magnetization to about $100 \mathrm{Oe}$, allowing the observation of a bistable remanent state. We observed current-induced free-layer reversal and free-layer excitations of which depend linearly on applied field. The evolution of those excitations with current and field provide a means to measure the individual dipolar field contributions from the reference layers and determine the change in the current polarization with the relative alignment of the reference magnetic layers. While the critical current for reversing the free layer is similar to previous $[\mathrm{Co} / \mathrm{Ni}]$-multilayer devices, we find the synthetic AF reference layer is insensitive to applied currents making them suitable for spin-torque device applications.

${ }^{1}$ B. N. Engel, J. Akerman, B. Butcher, R. W. Dave, M. DeHerrera, M. Durlam, G. Grynkewich, J. Janesky, S. V. Pietambaram, N. D. Rizzo, J. M. Slaughter, K. Smith, J. J. Sun, and S. Tehrani, IEEE Trans. Magn. 41, 132 (2005).

${ }^{2}$ W. J. Gallagher and S. S. P. Parkin, IBM J. Res. Dev. 50, 5 (2006).

${ }^{3}$ J. A. Katine and E. E. Fullerton, J. Magn. Magn. Mater. 320, 1217 (2008).

${ }^{4}$ J. M. Slaughter, Annu. Rev. Mater. Res. 39, 277 (2009).

${ }^{5}$ W.S. Zhao, E. Belhaire, C. Chappert, and P. Mazoyer, ACM Transactions on Embedded Computing Systems 9, 14 (2009).

${ }^{6}$ S. Mangin, D. Ravelosona, J. A. Katine, M. J. Carey, B. D. Terris, and E.

E. Fullerton, Nature Mater. 5, 210 (2006).

${ }^{7}$ S. Mangin, Y. Henry, D. Ravelosona, J. A. Katine, and E. E. Fullerton, Appl. Phys. Lett. 94, 012502 (2009).

${ }^{8}$ H. Meng and J. P. Wang, Appl. Phys. Lett. 88, 172506 (2006).

${ }^{9}$ T. Seki, S. Mitani, K. Yakushiji, and K. Takanashi, Appl. Phys. Lett. 88 172504 (2006).

${ }^{10}$ M. Nakayama, T. Kai, N. Shimomura, M. Amano, E. Kitagawa, T. Nagase, M. Yoshikawa, T. Kishi, S. Ikegawa, and H. Yoda, J. Appl. Phys. 103, 07A710 (2008)

${ }^{11}$ J. C. Slonczewski, J. Magn. Magn. Mater. 159, L1 (1996).

${ }^{12}$ J. R. Childress and R. E. Fontana, C. R. Phys. 6, 997 (2005).

${ }^{13}$ S. S. P. Parkin, N. More, and K. P. Roche, Phys. Rev. Lett. 64, 2304 (1990).

${ }^{14}$ N. C. Emley, F. J. Albert, E. M. Ryan, I. N. Krivorotov, D. C. Ralph, R. A. Buhrman, J. M. Daughton, and A. Jander, Appl. Phys. Lett. 84, 4257 (2004).

${ }^{15}$ J. A. Katine, F. J. Albert, R. A. Buhrman, E. B. Myers, and D. C. Ralph, Phys. Rev. Lett. 84, 3149 (2000).

${ }^{16}$ M. J. Carey, N. Smith, S. Maat, and J. R. Childress, Appl. Phys. Lett. 93 102509 (2008).

${ }^{17}$ N. Smith, S. Maat, M. J. Carey, and J. R. Childress, Phys. Rev. Lett. 101, 247205 (2008). 